\title{
EFFECTS OF CAPILLARITY ON MICROSCOPIC FLOW IN POROUS MEDIA
}

Progress Report

for Period June 1, 19y2 - May 31, 1993

Michael J. Miksis

Department of Engincering Sciences

and Applied Mathematics

Northwestern Universiy

Evanston, Illinois 60208

January 1993

Prepared for

THE U.S. DEPARTMENT OF ENERGY

AGREEMENT NO. DE-FG02-88ER13927

\section{DISCLAIMER}

\begin{abstract}
This report was prepared as an account of work sponsored by an agency of the United States Government. Neither the United States Government nor any agency thereof, nor any of their employees, makes any warranty, express or implied, or assumes any legal liability or responsibility for the accuracy, compieieitess, oi ujofuliness of any information, anparatus, product, or process disclosed, or represents that its use would not infringe privately owned rights. Reference herein to any specific commercial product, process, or service by trade name, trademark, manufacturer, or otherwise does not necessarily constitute or imply its endorsement, recommendation, of favoring by the United States Government or any agency thereof. The views and opinions of authors expreseed berein do not necessarily state or reflect those of the United Strtes Government or any agency thereof.
\end{abstract}




\section{Abstract}

The central theme of this proposal is to study the effects of capillarity on the motion of a fluid interface and to apply these results to flow in porous media. Here we report on several problems considered this year, the second year of the grant. In particular we have developed a numerical code to study the dynamics of a gas bubble in a pore in order to examine the fundamental mechanism for the generation of a foam in a porous material, we have started an investigation of the stability of a foam lamella in order to understand the stability of foam flow in a porous material and we have derived systematically a slip coefficient for flow over a rough surface, e.g., as in a pore. In addition we report on work on several other problems. 
Several projects have been under investigation during the second year of this grant. In the following I will be specific about the work.

The central theme in this proposal is to study the effects of capillarity on the motion of a fluid interface and to use these results to help uncierstand flow in porous media. During this year we have studied several problems in this direction. For example in collaboration with my graduate student, J. Tsai, we have studied the motion of a fluid interface on the microscopic scale in a porous media, e.g., in a pore. This work is the Ph.D. thesis of J. Tsai which will be completed by September 1993. We are currently studying how a gas bubble moves inside of a pore with non-parallel walls in a pressure driven flow. The purpose of this investigation is to understand how a foam is formed. This foam formation process is called 'snap off' in the literature and is not completely understood. We have been studing this problem computationally and during this year a computer code has been developed. The numerical solution of this free boundary problem consists of solving the Stokes equations for the liquid within the pore by using a boundary intergal method. We have considered the motion of bubbles and drops (we will always use the word bubble with the understanding that the results apply to either a bubble or drop) in both straight-sided and constricted capillaries. In the straight-sided cases, a bubble moving in a capillary can either aprroach a steady state shape where the back is flatter than the front, egg shape, or it can become unstable and a jet of liquid can enter the back of the bubble and shoot through the front. These cases are illustrated in Figures 1 and 2 where we show a time sequence of shapes. The difference between the two cases is that the Capillary number is higher in the unsteady case. The constricted tube case is more interesting for applications since it better illustrates a pore where the geometry is more variable. Here we have identified three possible effects as the bubble passes through the constriction. First, fig. 3, the bubble can just squeeze through. Second, fig. 4, if we increase the Capillary number we find that as the bubble is passing through the constriction, an instability can be initiated where a jet of liquid from the back moves toward the front and breaks the bubble up and hence forms a foam, i.e., smaller bubbles. Finally, fig. 5, the third possibility is that as the bubble begins to pass through the constriction, another type of instability is observed where part of the bubble 'snaps' off and hence forms a foam. This was generated by lowering the slope of the constriction. These results imply that there are three ways that a foam can be formed: by the snap-off process, by the jet process and by a surface tension instability which occurs as a thin film is driven along the capillary wall (not illustrated here). We have been doing an extensive investigation of how the Capillary number, viscosity ratio of the two fluids, the gap width, and the slope of the constriction effect the dynamics of the bubble. The results compare well with experiments in the literature. This is the first extensive investigation of the generation of a foam.

As can be seen from the above results, a foam can have a wide range of bubble sizes, and still be called a foam. For example, one could have many small bubbles or few very large bubbles. The latter, where the bubble fills up a capillary except for a thin film along the wall and a thin lamella region which separates two bubbles, would be the more useful case since it would produce a mixture with a higher effective viscosity and therefore can be expected to be a more stable means for 
enhanced oil recovery or for cleaning up hazardous waste. Hence we are investigating the stability of a thin lamella as it is driven through a capillary. This work is being done in collaboration with my graduate student, Michael P. Ida, who started working with me in September of 1992. Our current model of the lamella motion replaces the plateau boarder, which connects the lamella region with the thin film coating the walls, by a contact line. Hence the problem reduces to studing the pressure driven motion of a thin lamella inside of a capillary tube where the conditions on the liquid at the wall are governed by a slip condition and a contact angle - slip velocity condition. The aim is to study the stability and dynamics of the lamella. We started our analysis by coinsidering a 2-D free film. The evolution equations for the thin film were solved numerically (a pseudospectral method) The results were compared to an weakly nonlinear analytical result to find good comparsion in some limits and bad in others for the time for the film to break. In fig. 6 we plot a time sequences from an initial perturbation for the breaking of a symmetric free film. The final plot is almost at breaking. In fig. 7 we plot the minimum thickness of the film as a function of time and compare a numerical and analytical result. We see that there is about a $25 \%$ difference between the two (the small parameter in this analysis was 0.1 ). $\Lambda$ linear stability analysis for both the free film case and the case of a film trapped between two walls gives the stability result that if the IIamaker constant is sufficiently larger than the surface tension (times a constant depending on the geometry and other physical parameters) then the film becomes unstable. This result has been generalized to $3-\mathrm{D}$ and we are currently studing the motion of a non-symmetric lamella in a capillary tube with the effect of the contact line included.

We have also been considering several other problems. For example we have considered the motion of a fluid over a rough wall. In the limit where the scale of the roughness is the same as the amplitude of the roughness, a slip law can be derived by an asymptotic analysis. Previous derivations required an explicit form of the roughness. Extensions to other limits of the roughness are now being done.

We have also been considering the problem of a coating flow along an inclined plane. This is an extension of a recently accepted article (Journal of Fluid Mechanics) of mine with L. Hocking of the University College London. The current work involves studing the stability of a semi-infinite sheet of liquid as it coats an inclined piane where we include the effect of the contact line plus van der Waal forces. This model is interesting since it unites the microscopic forces with the behavior of the contact line. We are currently solving the linear stability problem numerically. This result should give insite into the stability of a driven contact line, as in foam flow in a porous material.

When an acoustic wave strikes an elastic panel the wave is scattered off plus the panel vibrates. The standard approach is to solve the equations of motion of the liquid coupled with the elastic equations by a numerical method. This is generally a difficult task. By using the fact that the wave speed of the acoustic wave is much smaller than the speed of propagation in the elastic panel, a reduced system of equations describing the dynamics of the panel can be systematically derived via an asymptotic analysis. The reduced set of equations involves solving the equations for the cvolution of the planel driven by a new inhomogeneou: term involving the incident acoustic wave. 
This is a great simplification of the problem since the equations in the liquid do not need to be solved. Ilence a three dimensional problem reduces to a two dimensional problem. The calculation was done jointly with L. Ting of the Courant Institute of New York University.

During this period I have taken one summer month in salary plus $10 \%$ academic year. I plan to take two summer months in salary during $93-94$. 


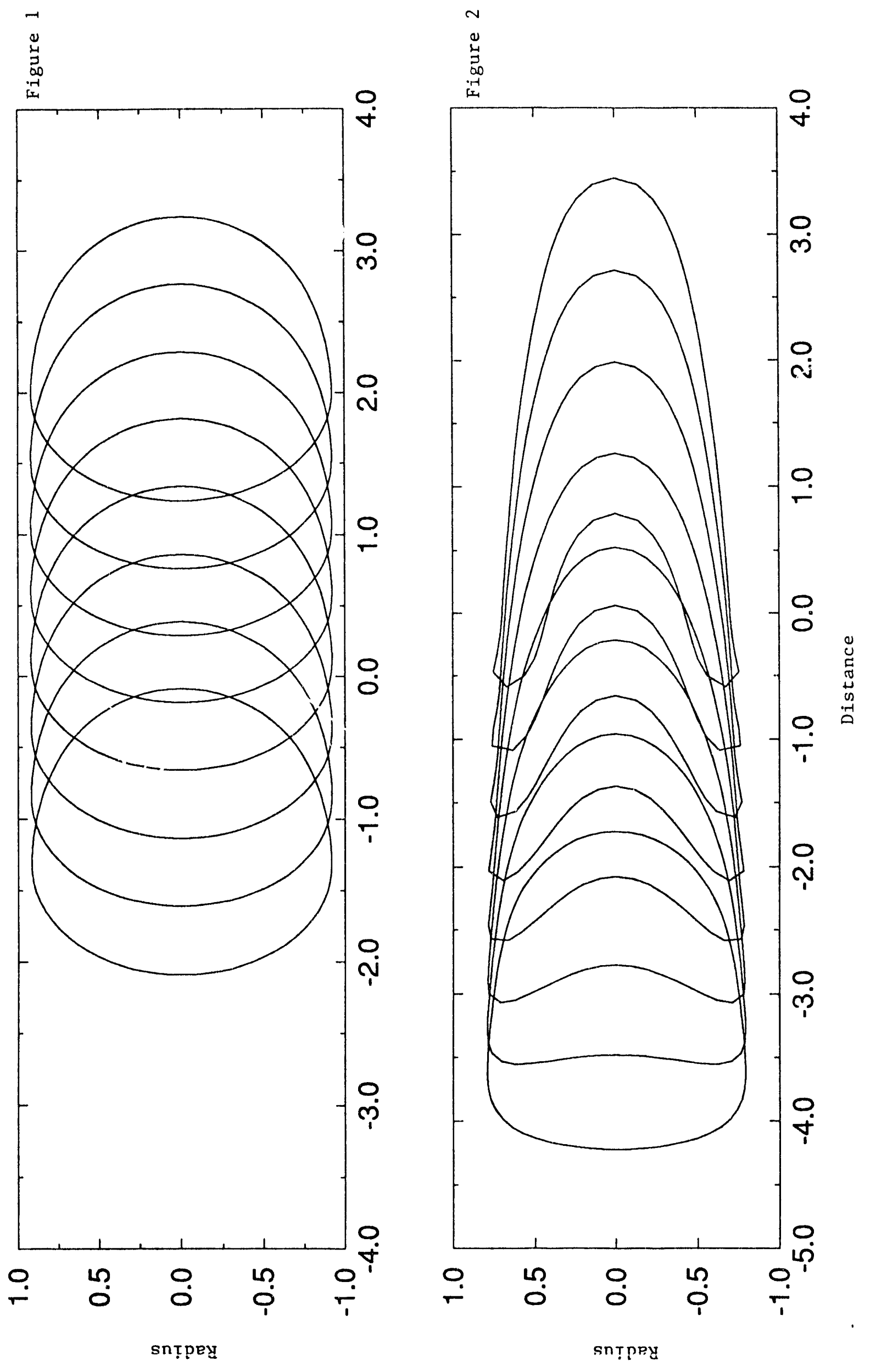




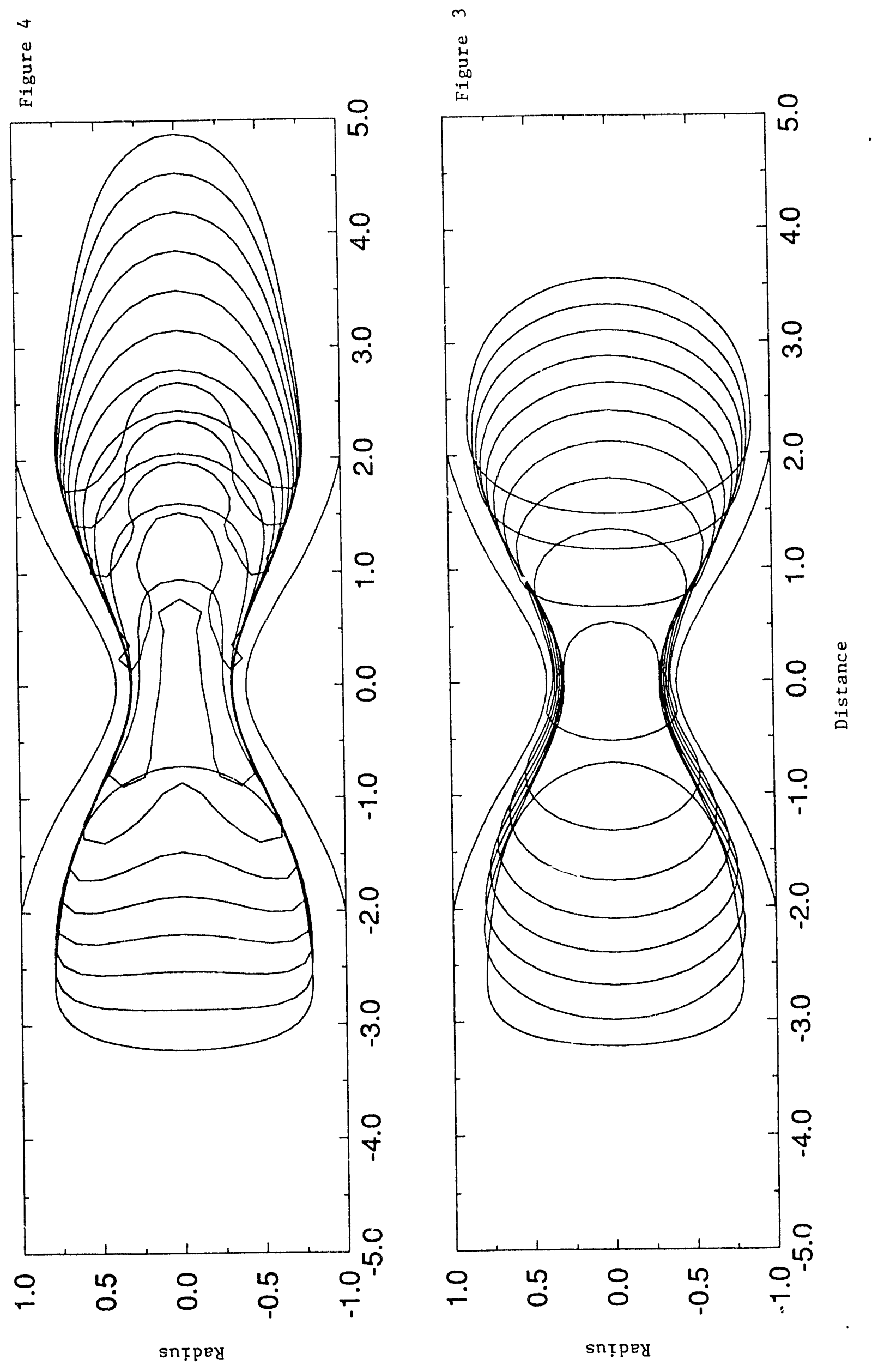


n

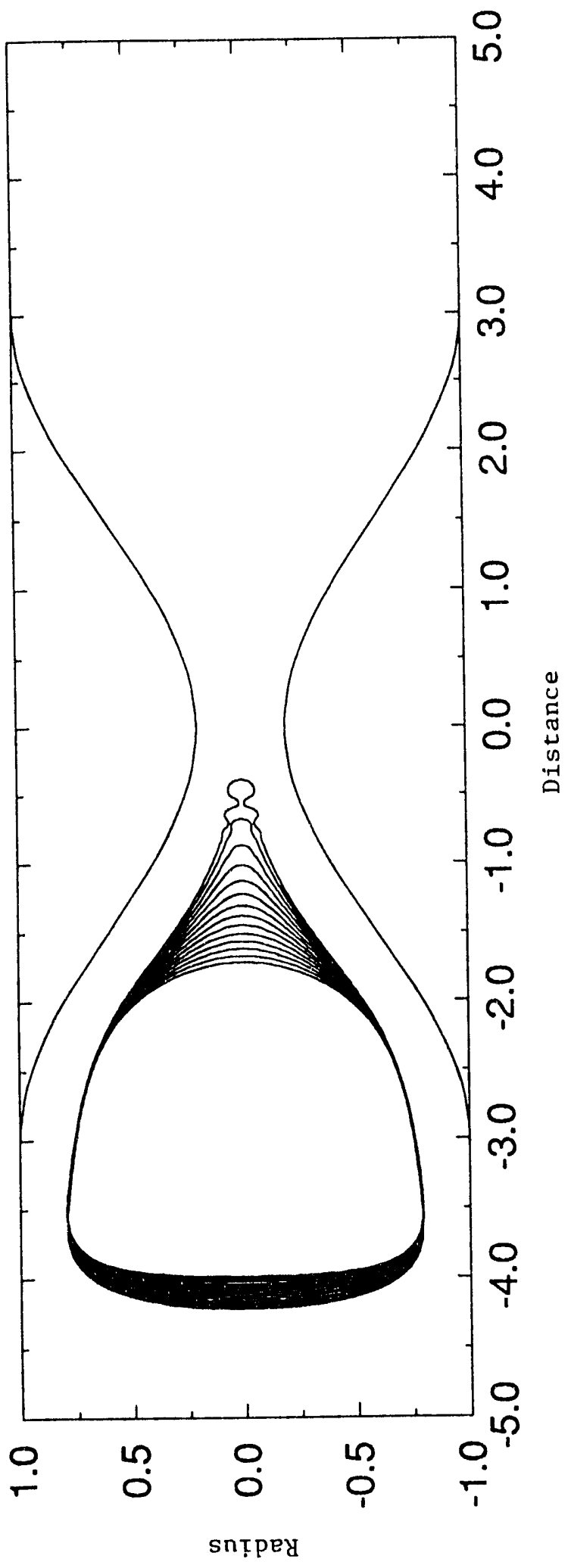




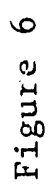

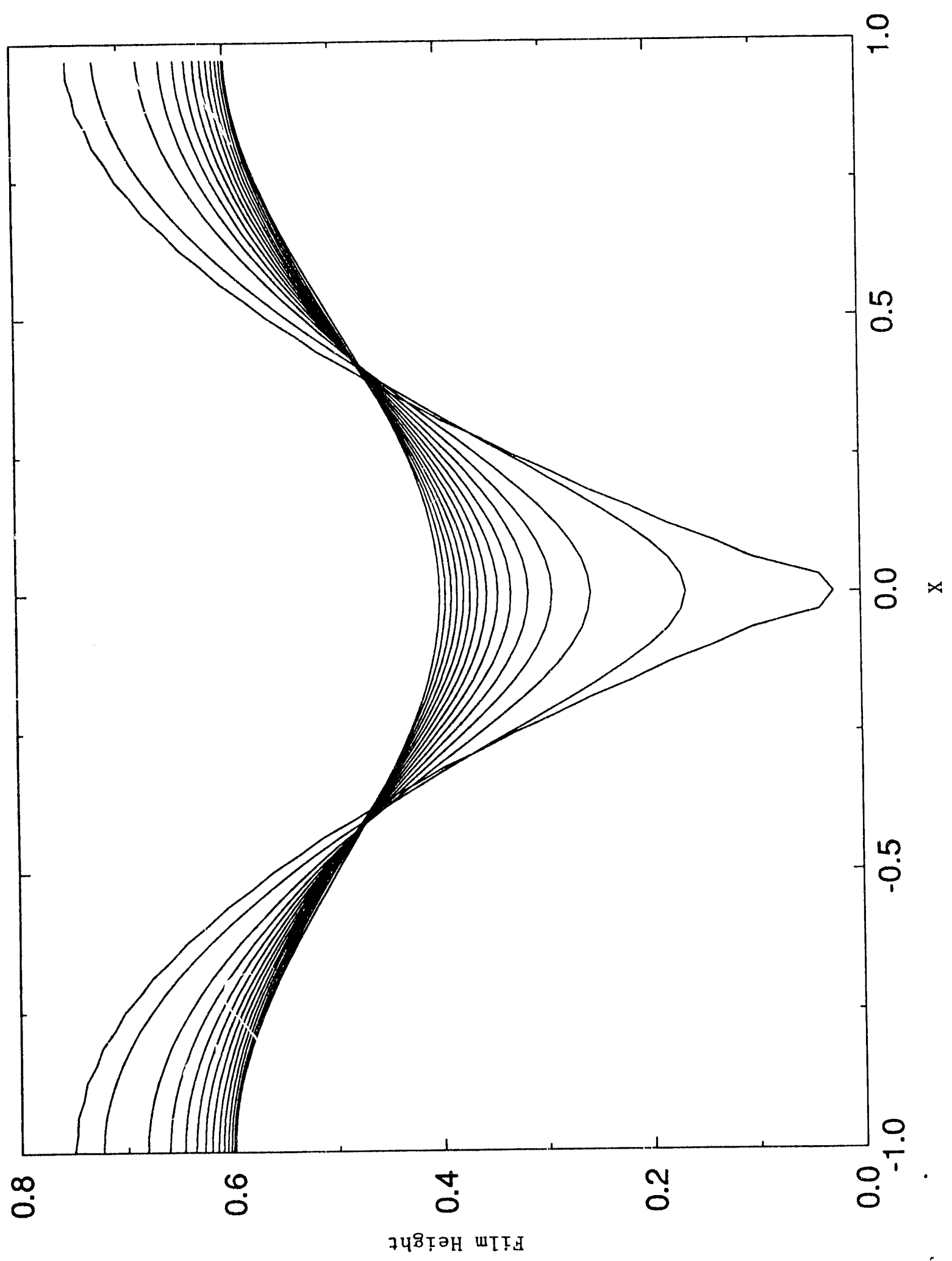




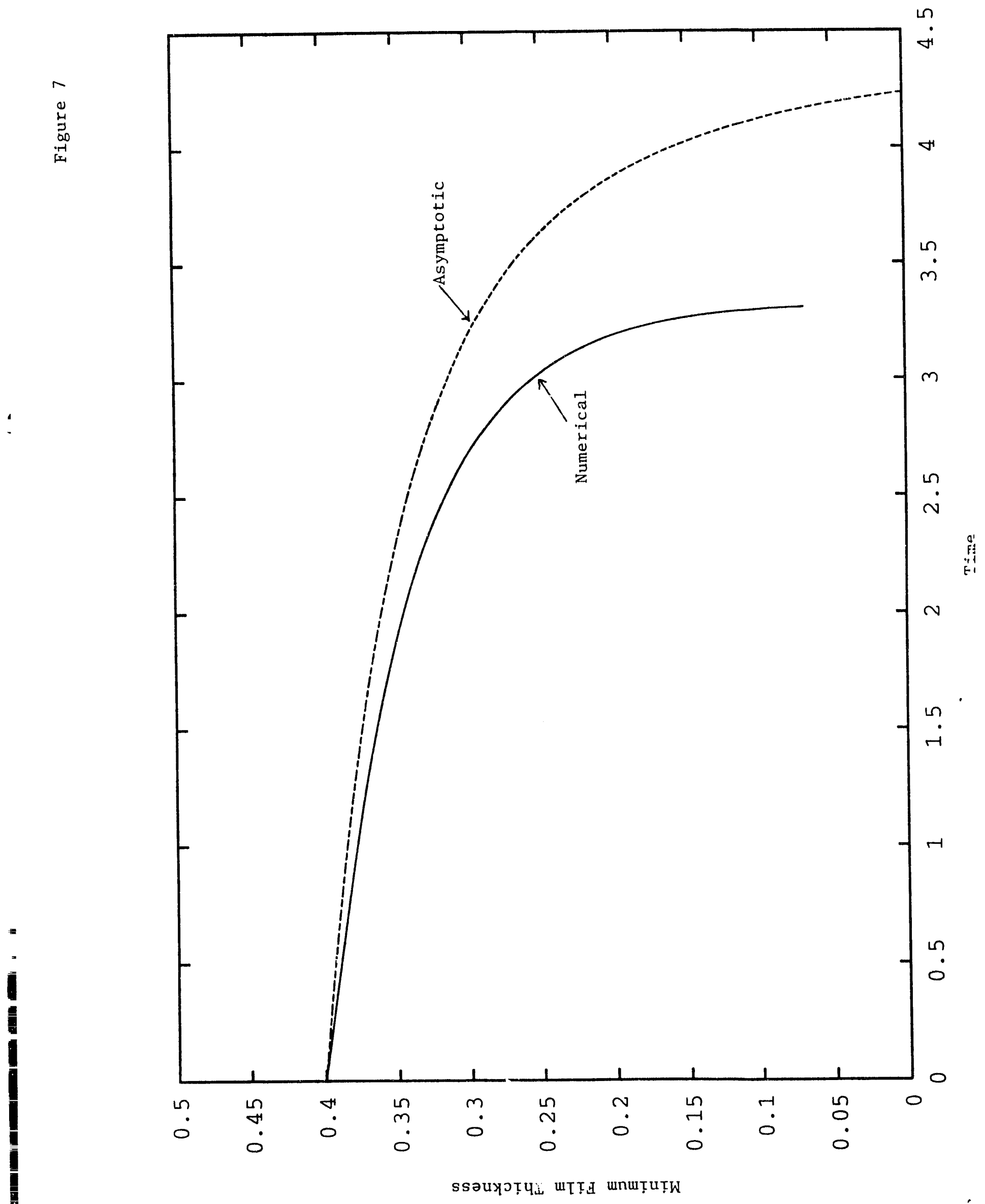



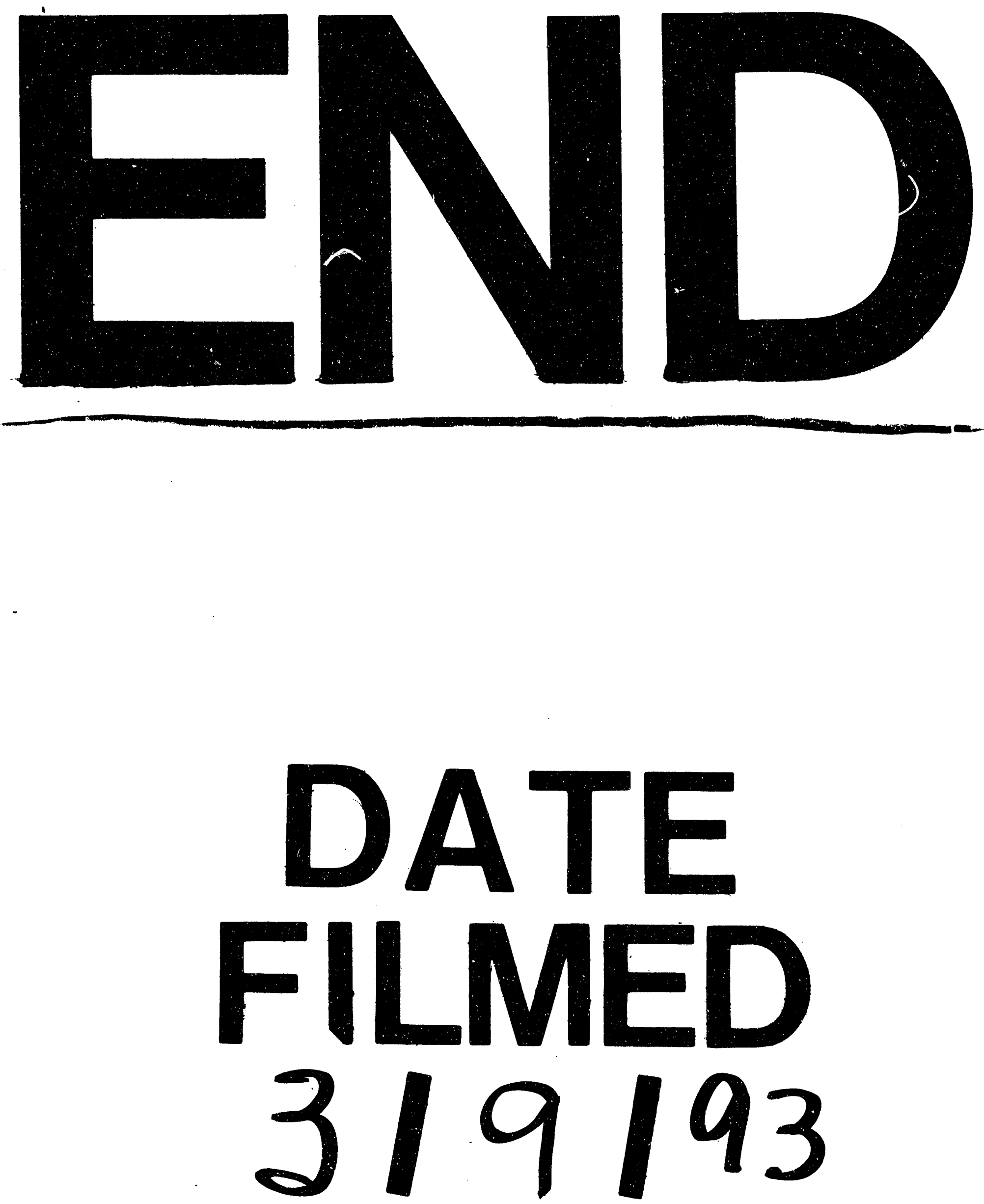
